\title{
Inter-hemispheric Functional Connections Are More Vulnerable to Attack Than Structural Connection in Patients With Irritable Bowel Syndrome
}

\begin{abstract}
Guangyao Liu, ${ }^{1}$ Shan Li, ${ }^{2}$ Nan Chen, ${ }^{2}$ Ziyang Zhao, ${ }^{2}$ Man Guo, ${ }^{2}$ Hong Liu, ${ }^{1}$ Jie Feng, ${ }^{3}$ Dekui Zhang, ${ }^{3 *}$ Zhijun Yao, ${ }^{2 *}$ and Bin Hu ${ }^{2,4,5,6 *}$
${ }^{1}$ Department of Magnetic Resonance, Lanzhou University Second Hospital, Lanzhou, China; ${ }^{2}$ Gansu Provincial Key Laboratory of Wearable Computing, School of Information Science and Engineering, Lanzhou University, Lanzhou, China; ${ }^{3}$ Department of Gastroenterology, Lanzhou University Second Hospital, Lanzhou, China; ${ }^{4}$ CAS Center for Excellence in Brain Science and Intelligence Technology, Shanghai Institutes for Biological Sciences, Chinese Academy of Sciences, Shanghai, China; ${ }^{5}$ Joint Research Center for Cognitive Neurosensor Technology of Lanzhou University and Institute of Semiconductors, Chinese Academy of Sciences, Lanzhou, China; and ${ }^{6}$ Engineering Research Center of Open Source Software and Real-Time System (Lanzhou University), Ministry of Education, Lanzhou, China
\end{abstract}

\section{Background/Aims}

Irritable bowel syndrome (IBS) is a prevalent functional gastrointestinal disease characterized by recurrent abdominal pain and bowel dysfunction. However, the majority of previous neuroimaging studies focus on brain structure and connections but seldom on the inter-hemispheric connectivity or structural asymmetry. This study uses multi-modal imaging to investigate the abnormal changes across the 2 cerebral hemispheres in patients with IBS.

\section{Methods}

Structural MRI, resting-state functional MRI, and diffusion tensor imaging were acquired from 34 patients with IBS and 33 healthy controls. The voxel-mirrored homotopic connectivity, fractional anisotropy, fiber length, fiber number, and asymmetry index were calculated and assessed for group differences. In addition, we assessed their relevance for the severity of IBS.

\section{Results}

Compared with healthy controls, the inter-hemispheric functional connectivity of patients with IBS showed higher levels in bilateral superior occipital gyrus, middle occipital gyrus, precuneus, posterior cingulate gyrus, and angular gyrus, but lower in supplementary motor area. The statistical results showed no significant difference in inter-hemispheric anatomical connections and structural asymmetry, however negative correlations between inter-hemispheric connectivity and the severity of IBS were found in some regions with significant difference.

\section{Conclusions}

The functional connections between cerebral hemispheres were more susceptible to IBS than anatomical connections, and brain structure is relatively stable. Besides, the brain areas affected by IBS were concentrated in default mode network and sensorimotor network.

(J Neurogastroenterol Motil 2021;27:426-435)

\section{Key Words}

Brain; Default mode network; Irritable bowel syndrome; Neuroimaging 


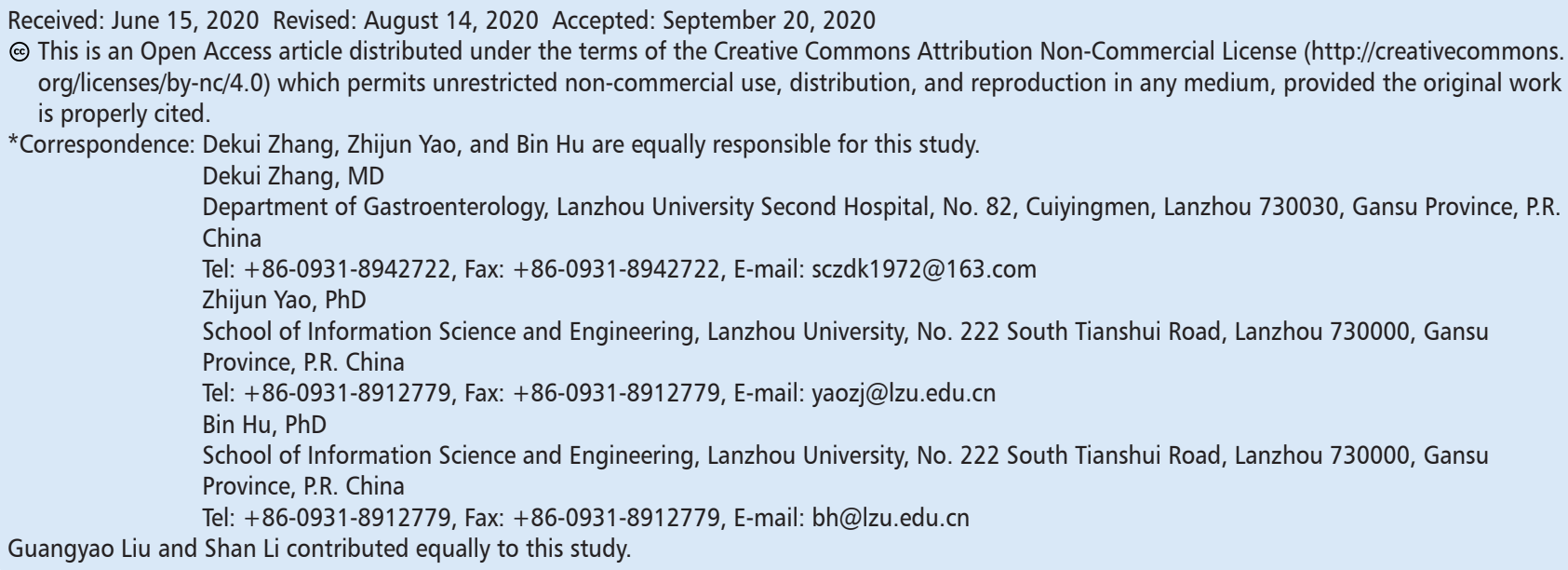

\section{Introduction}

Irritable bowel syndrome (IBS) is a common functional gastrointestinal disease which is characterized by abdominal pain and disordered defecation. ${ }^{1}$ Global studies that recorded the prevalence of IBS is about $5-22 \%{ }^{2}$ and this number is about $5-10 \%$ in China. ${ }^{3,4}$ In the general population, IBS is more prevalent in women compared with men. ${ }^{5}$ The subtypes of IBS were distinguished depend on predominant stool pattern, including constipation-predominant IBS, diarrhea-predominant IBS, mixed IBS alternating constipation and diarrhea, and an unsubtyped IBS. The pathogenesis of IBS appears to be multifactorial, including diet, an altered neuroendocrine system, abnormal intestinal microbiota, genetics, and mucosal low-grade inflammation. ${ }^{6}$ Hitherto, the underlying pathophysiological mechanisms of IBS are still incompletely understood.

The majority of IBS patients were discovered with psychological abnormalities, such as fatigue, anxiety, and depression. ${ }^{7-10}$ The human gut and brain interact complexly through the gut-brain axis (GBA), which includes the central nervous system, autonomic nervous system, enteric nervous system, and hypothalamic pituitary adrenal (HPA) axis. ${ }^{11}$ The enteric microbiota is widely distributed in the human gastrointestinal tract, although individuals' gut microbiota may not be identical, the relative abundance and distribution of these bacterial phylotypes are similar to healthy controls (HCs). IBS is considered an exemplary disorder of gut-brain communication. An increasing number of studies suggested that gut microbiota is an environmental factor that can regulate the brain through the GBA. ${ }^{12,13}$ In recent years, some studies have reported a relationship between neuropsychiatric and GBA, ${ }^{14-16}$ and suggested that the modulation of gut microbiota may be a viable strategy for the treat- ment of mental diseases. ${ }^{13}$

Noninvasive neuroimaging has become a popular method to identify neurophysiologic abnormalities in several diseases of the brain. Meanwhile, multimodal brain imaging has promoted a better understanding of brain diseases. Previous studies have reported significant brain abnormalities in patients with pain or mental diseases. ${ }^{17-19}$ For example, abnormal rest-state functional connectivity (rsFC) was reported in adults with major depressive disorders. ${ }^{20}$ Stress-related mental disorders such as major depressive disorders and anxiety disorders are associated with the change of brain structure, ${ }^{21}$ and over time these diseases can lead to more severe changes in the brain. Simultaneously, different types of chronic pain showed unique anatomical reorganization of brain. ${ }^{22}$

The human brain is remarkably asymmetrical and which increases throughout life. ${ }^{23}$ Despite the left and right halves of the brain resembling each other, the 2 hemispheres also display significant anatomical and functional differences. ${ }^{24}$ There is an advantage to bihemispheric processing, provided that each hemisphere participates in specific functions. Besides, the interaction of perception, cognition, and behavior between 2 brain hemispheres is important for brain functioning. ${ }^{25}$ Several studies have suggested stress-related mental disorders displayed the disruption of inter-hemispheric structural connectivity and the loss of functional connectivity (FC), 26,27 accompanied by significant changes in structural asymmetry. ${ }^{28}$

Recently analyses have identified regional and network alterations in the brain of patients with IBS. ${ }^{29,30}$ Compared to HCs, alterations in rsFC of the default mode network (DMN), central executive network, and sensorimotor network have been observed in patients with IBS. ${ }^{31,32}$ A recent study reported IBS had lower fractional anisotropy (FA) and higher mean diffusivity in the thalamic regions, the basal ganglia, and the sensory/motor association/ 
integration regions. ${ }^{33}$ However, there is a lack of consistency with regard to the morphologic abnormalities of the brain reported in patients with IBS, ${ }^{34-36}$ which is probably influenced by the gender and IBS subtypes of patients. ${ }^{37,38}$ Although a number of researches on patients with IBS have investigated the abnormalities in specific brain areas and connections, few studies focus on the inter-hemispheric connections and asymmetry.

We hypothesized that patients with IBS would display aberrant inter-hemispheric functional and anatomical connections compared with healthy adults. Five types of MRI features, including voxelmirrored homotopic connectivity (VMHC) from functional MRI (fMRI), asymmetry index (AI) from structural MRI, FA, fiber length, and FN from diffusion tensor imaging (DTI), were analyzed in this study. To the best of our knowledge, this is the first attempt to evaluate the asymmetry and inter-hemispheric connectivity of patients with IBS.

\section{Materials and Methods}

\section{Participants}

Thirty-four participants with IBS (24 diarrhea-predominant IBS, 4 constipation-predominant IBS, and 6 mixed IBS) and 33 age-, gender-matched HCs were recruited from Lanzhou University Second Hospital (see Table 1 for details). All participants were right-hand. The diagnosis was based on the Rome IV criteria by

Table 1. Demographics and Psychological Assessments Between Irritable Bowel Syndrome and Healthy Controls

\begin{tabular}{lccl}
\hline & IBS $(\mathrm{n}=34)$ & $\mathrm{HC}(\mathrm{n}=33)$ & $P$-value \\
\hline Gender $(\mathrm{F} / \mathrm{M})$ & $16 / 18$ & $22 / 11$ & 0.105 \\
Age $(\mathrm{yr})$ & $27.35 \pm 4.40$ & $25.67 \pm 4.56$ & 0.128 \\
BMI $\left(\mathrm{kg} / \mathrm{m}^{2}\right)$ & $22.56 \pm 4.08$ & $20.36 \pm 2.24$ & 0.008 \\
Education $(\mathrm{yr})$ & $17.35 \pm 1.92$ & $17.00 \pm 2.02$ & 0.466 \\
IBS-SSS & $247.06 \pm 39.04$ & $120.00 \pm 23.98$ & $<0.001$ \\
IBS-QOL & $83.33 \pm 10.97$ & $96.88 \pm 3.57$ & $4.683 \mathrm{e}-9$ \\
PCS & $10.15 \pm 8.39$ & $2.24 \pm 4.51$ & $1.034 \mathrm{e}-5$ \\
PHQ-15 & $11.06 \pm 7.49$ & $0.00 \pm 0.00$ & $4.055 \mathrm{e}-12$ \\
HAMA & $10.82 \pm 7.79$ & $2.18 \pm 2.95$ & $1.074 \mathrm{e}-7$ \\
HAMD & $9.41 \pm 6.82$ & $2.82 \pm 4.82$ & $2.332 \mathrm{e}-5$ \\
SF-MPQ & $8.59 \pm 5.65$ & $0.00 \pm 0.00$ & $1.453 \mathrm{e}-12$ \\
\hline
\end{tabular}

IBS, irritable bowel syndrome; HC, healthy control; F, female; M, male; BMI, body mass index; IBS-SSS, IBS Severity Scoring System; IBS-QOL, IBS-quality of life; PCS, Pain Catastrophizing Scale; PHQ-15, Patient Health Questionnaire; HAMA, Hamilton Anxiety Scale; HAMD, Hamilton Depression Scale; SF-MPQ, short-form McGill Pain Questionnaire.

Values are expressed as $n$ or mean $\pm \mathrm{SD}$. a gastroenterologist who is experienced in the evaluation of IBS. ${ }^{39}$ Exclusion criteria included psychiatric disease, other severe disease, and history of drug or alcohol abuse. Written informed consent to participate was obtained from each participant. All procedures were in accordance with the guidelines. Ethical approval (2019A-176) was obtained from the medical ethics committee of Lanzhou University Second Hospital.

\section{Psychological Assessment}

All participants underwent a battery of standardized psychological assessments before MRI scanning. Body mass index $(\mathrm{BMI}){ }^{40}$ IBS Severity Scoring System (IBS-SSS, range $\left.=100-500\right),{ }^{41}$ Pain Catastrophizing Scale $(\mathrm{PCS}$, range $=0-52),{ }^{42}$ Patient Health Questionnaire (PHQ-15, range $=0-30),{ }^{43}$ and short-form McGill Pain Questionnaire (SF-MPQ, range $=0-60)^{44}$ were used to evaluate the severity of health problems. Anxiety and depression states were measured using the Hamilton Anxiety Scale (HAMA, range $=0-56)^{45}$ and Hamilton Depression Scale (HAMD, range $=$ $0-54){ }^{46}$ IBS-quality of life (IBS-QOL, range $=0-100$ ) assessed the influence of bowel habit on daily life. ${ }^{47} \mathrm{BMI}$, IBS-SSS, PCS, PHQ-15, SF-MPQ, HAMA, and HAMD assessed the level of obesity, severity of IBS symptoms, degree of pessimism about pain, somatic symptom burden, overall intensity of pain, and severity of anxiety and depression, respectively. The higher score means the higher level of these symptoms. Besides all that, IBS-QOL is a valuable scale to reflect the impact of physical and psychological in IBS patients, and the higher score indicate better QOL.

\section{Image Acquisition}

All MRI data were acquired on a 3.0 T Siemens scanner using the standard 8-channel head coil at Lanzhou University Second Hospital. High-resolution 3-dimensional T1-weighted data were acquired with the following parameters: repetition time $(\mathrm{TR})=2000 \mathrm{msec}$, echo time $(\mathrm{TE})=2.67 \mathrm{msec}$, voxel size $=1.0 \times$ $1.0 \times 1.0 \mathrm{~mm}$, slice thickness $=1.0 \mathrm{~mm}$, field of view $(\mathrm{FOV})$ read $=256 \mathrm{~mm}, \mathrm{FOV}$ phase $=87.5 \%$, flip angle $=12^{\circ}$, inversion time $(\mathrm{TI})=900 \mathrm{msec}$. All of the images were checked to rule out structural abnormalities. Resting-state fMRI images were acquired using an echo-planar-imaging sequence with the following parameters: $\mathrm{TR}=2000 \mathrm{msec}, \mathrm{TE}=30 \mathrm{msec}$, voxel size $=3.4 \times 3.4 \times$ $3.0 \mathrm{~mm}$, slice thickness $=3.0 \mathrm{~mm}$, FOV read $=220 \mathrm{~mm}$, FOV phase $=100.0 \%$, flip angle $=90^{\circ}$. DTI images were performed with echo-planar-imaging sequence with the following parameters: $\mathrm{TR}=7600 \mathrm{msec}, \mathrm{TE}=90 \mathrm{msec}$, voxel size $=2.0 \times 2.0 \times 3.0 \mathrm{~mm}$, slice thickness $=3.0 \mathrm{~mm}$, FOV read $=256 \mathrm{~mm}, \mathrm{FOV}$ phase $=$ 
$100.0 \%$, Throughout scans, all subjects were instructed to relax, keep their eyes closed and avoid thinking of anything in particular.

\section{Data Preprocessing}

Functional MRI preprocessing was performed using the data processing assistant for resting-state $\mathrm{fMRI}^{48}$ with statistical parametric mapping (https://www.fil.ion.ucl.ac.uk/spm). ${ }^{49}$ The first 10 images of each functional time series were discarded to allow signal stabilization and adaptation of the participants to the scanning environment. Slice timing correction, head motion correction, spatial normalization to the Montreal Neurological Institute template, and spatially smoothed with $6 \mathrm{~mm}$ full width at half maximum Gaussian kernel were applied to the remaining volumes. Subsequently, linear detrending and band-pass-filter $(0.01-0.08 \mathrm{~Hz})$ were performed to reduce the effect of low-frequency drift and high-frequency physiological noise. Nuisance covariates including 6 head motion parameters, cerebrospinal fluid signals, global mean, and white matter were removed by linear regression.

The 3-dimensional T1 images were processed using the FreeSurfer software (http://surfer.nmr.mgh.harvard.edu), which include a set of automatic sequences to reconstruct the surface of whole brain. ${ }^{50,51}$ Details of this pipeline were described in previous studies. ${ }^{50,52}$ A 2-dimensional cortical surface was calculated and we divided the whole brain into 45 anatomically labeled areas for each hemisphere using the Automated Anatomical Labeling template.

For the DTI data, we first converted the format of images from DICOM into 4-dimensional nifti-1 format file. Data processing was executed by using the pipeline for analyzing brain diffusion images (PANDA; http://www.nitrc.org/projects/panda/), ${ }^{53}$ which is based on FSL (http://fsl.fmrib.ox.ac.uk/fsl) ${ }^{54}$ and Diffusion Toolkit. ${ }^{55}$ The approach of processing included correction for simple head motion and eddy current distortions using affine transformation to the b0 image. After correction, we obtained the FA maps of each subject. Fiber assignment by continuous tracking algorithm was subsequently performed to obtain the whole-brain fiber tractography. Path tracking procedure started from the deep white matter regions and terminated at a voxel with a turning angle greater than $45^{\circ}$ or reached a voxel with an FA of $<0.2$. The DTI metrics were calculated for each subject, followed by spatial standardization and Gaussian smoothing.

\section{Calculation of Voxel-mirrored Homotopic Connectivity}

The VMHC was obtained to quantify the rsFC between each voxel in one hemisphere and its mirrored counterpart in the opposite hemisphere. ${ }^{56}$ We computed the value of VMHC using resting-state fMRI data analysis toolkit (REST V1.8; http://www. restfmri.net/forum/REST_V1.8). ${ }^{57}$ The procedure of computation included the follow steps: (1) creating a mean normalized T1 image and averaged it with left-right mirrored version to generate a group-specific symmetrical template, (2) registering the $\mathrm{T} 1 \mathrm{im}-$ age of each subject to the symmetrical template and applying this transformation to the corresponding functional data, (3) computing the Pearson's correlation coefficient between each voxel and its symmetrical interhemispheric counterpart, (4) applying the Fisher's $z$ transformation to improve normality. The resultant values constituted the VMHC maps and were used in group-level analyses.

\section{Voxel Based Morphometry-based Volumetric Analysis}

For each subject, the volume of each region of interest (ROI) and intracranial volume were obtained with FreeSurfer software (https://www.freesurfer.net/). To evaluate the possible structural abnormalities of hemispheric asymmetry produced by IBS, we calculated the AI using the following formula:

$$
\mathrm{AI}=[(\mathrm{L}-\mathrm{R}) /(\mathrm{L}+\mathrm{R})] / 2
$$

where $\mathrm{L}$ and $\mathrm{R}$ represent the corresponding volume on the left and right hemisphere respectively. The absolute value of AI ranges from 0.0 to 0.5 . Thus, positive and negative AI values indicate leftward (left larger than right) and rightward asymmetry respectively.

\section{Statistical Methods}

Statistical analyses were performed using REST V1.8. To test the group differences in age, years of education, psychological assessments, FA, FN, and fiber length, data was analyzed with Student's $t$ test. The chi-square test was employed to compare gender distributions between groups. A value of $P<0.05$ was considered significant. To examine the differences in AI between IBS and HCs, we used one-way ANOVA with intracranial volume as covariate. Individual-level VMHC maps were entered into a group-level voxel-wise $t$ test analysis. AlphaSim was used to correct for multiple comparisons, and significance threshold was defined as $P<0.05$. Lastly, Pearson correlation analyses were performed to assess the relationships between metrics and the severity of IBS. Significant correlation was defined as $r>0.3$ and $P<0.05$. 


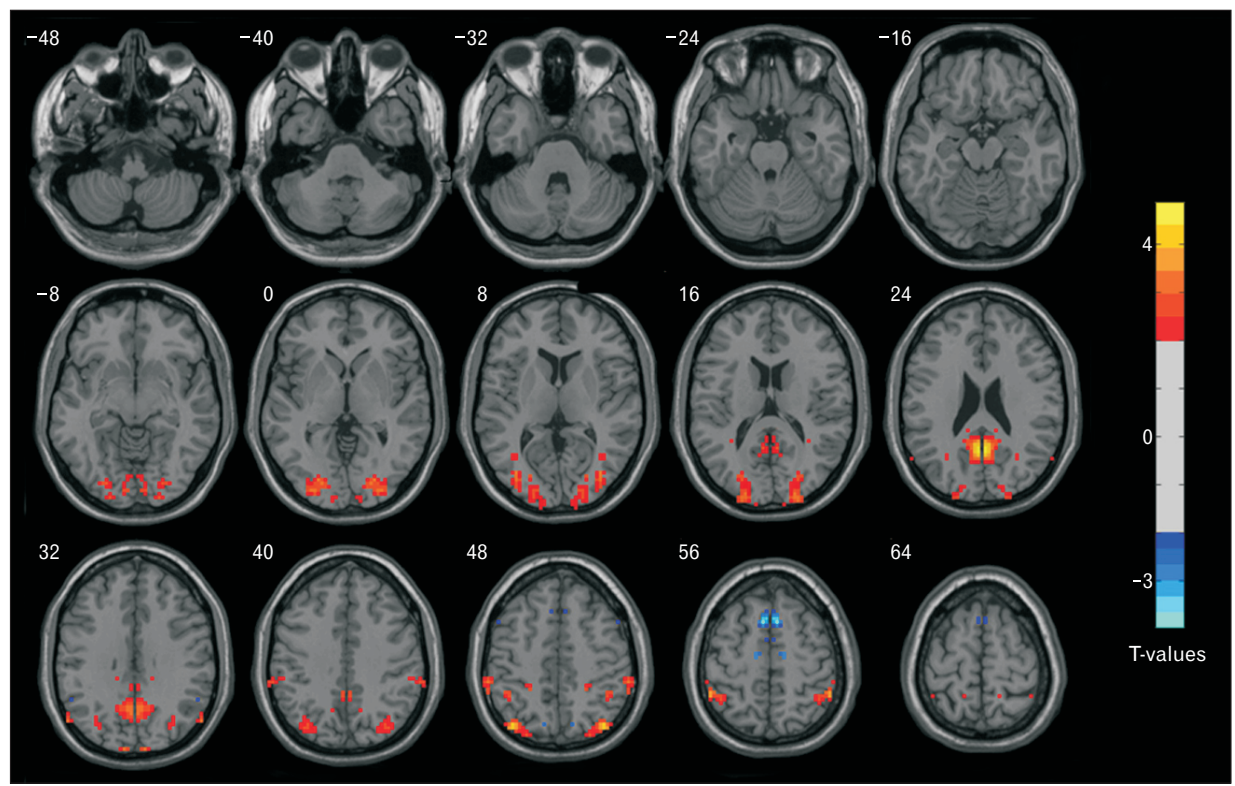

Figure 1. Statistical maps showing voxel-mirrored homotopic connectivity (VMHC) differences between irritable bowel syndrome (IBS) and healthy controls (HCs; $P<0.05$, corrected with Alphasim). IBS patients showed much higher VMHC in superior occipital gyrus, middle occipital gyrus, precuneus, posterior cingulate gyrus, and angular gyrus (red), while lower in supplementary motor area (blue). The color bar indicates the T-value from $t$ test between groups.

Table 2. Regions With Significant Differences in Voxel-mirrored Homotopic Connectivity $(P<0.05$, AlphaSim Corrected $)$

\begin{tabular}{lcccr}
\hline \multirow{1}{*}{\multicolumn{1}{c}{ Brain region }} & \multicolumn{3}{c}{$\begin{array}{c}\text { Peak MNI } \\
\text { coordinate }\end{array}$} & $\begin{array}{c}\text { Peak } \\
\end{array}$ \\
\cline { 2 - 4 } & $\mathrm{x}$ & $\mathrm{y}$ & $\mathrm{z}$ & \\
\hline Bilateral superior occipital gyrus & \pm 24 & -99 & 18 & 3.678 \\
Bilateral middle occipital gyrus & \pm 24 & -99 & 20 & 2.903 \\
Bilateral precuneus & \pm 3 & -57 & 27 & 4.882 \\
Bilateral posterior cingulate gyrus & \pm 3 & -57 & 29 & 4.854 \\
Bilateral supplementary motor area & \pm 6 & 15 & 57 & -3.998 \\
Bilateral angular gyrus & \pm 39 & -75 & 45 & 4.327 \\
\hline
\end{tabular}

MNI, Montreal Neurological Institute.

\section{Results}

\section{Characteristics of Participants}

The demographics and psychological assessments of participants were presented in Table 1 . There was no significant difference in gender, age or education level between IBS patients or HC adults. IBS patients had a significant higher BMI, IBS-SSS, PCS, PHQ-15, HAMA, HAMD, and SF-MPQ scores than that in HCs, whereas IBS-QOL declined significantly.
Table 3. Correlation Analyses Between Voxel-mirrored Homotopic Connectivity and Irritable Bowel Syndrome Severity Scoring System Score

\begin{tabular}{cccccc}
\hline \multirow{2}{*}{ Brain region } & \multicolumn{3}{c}{ Peak MNI } & Peak \\
& \multicolumn{3}{c}{ coordinate } & realue \\
\cline { 2 - 4 } & $\mathrm{x}$ & $\mathrm{y}$ & $\mathrm{z}$ & \\
\hline Bilateral middle occipital gyrus & \pm 45 & -87 & 15 & -0.600 \\
\hline
\end{tabular}

MNI, Montreal Neurological Institute.

\section{Functional Connectivity Differences Between Groups}

Figure 1 and Table 2 show the group comparisons of VMHC values. Patient with IBS had higher VMHC than the control group in the superior occipital gyrus (SOG), middle occipital gyrus (MOG), precuneus, posterior cingulate gyrus (PCG), and angular gyrus (ANG). Supplementary motor area (SMA) exhibited lower VMHC in the IBS than HC $(P<0.05$, Alphasim correction).

We also examined the correlation between $\mathrm{VMHC}$ in each pair of ROIs and IBS-SSS scores, but a significant negative correlation between VMHC and the severity of IBS were found only in the bilateral middle occipital gyrus $(r=-0.600, P<0.05)$ (Table 3$)$.

\section{Anatomical Connection Differences Between Groups}

We performed a between-group analysis for the AI, FA, fiber 

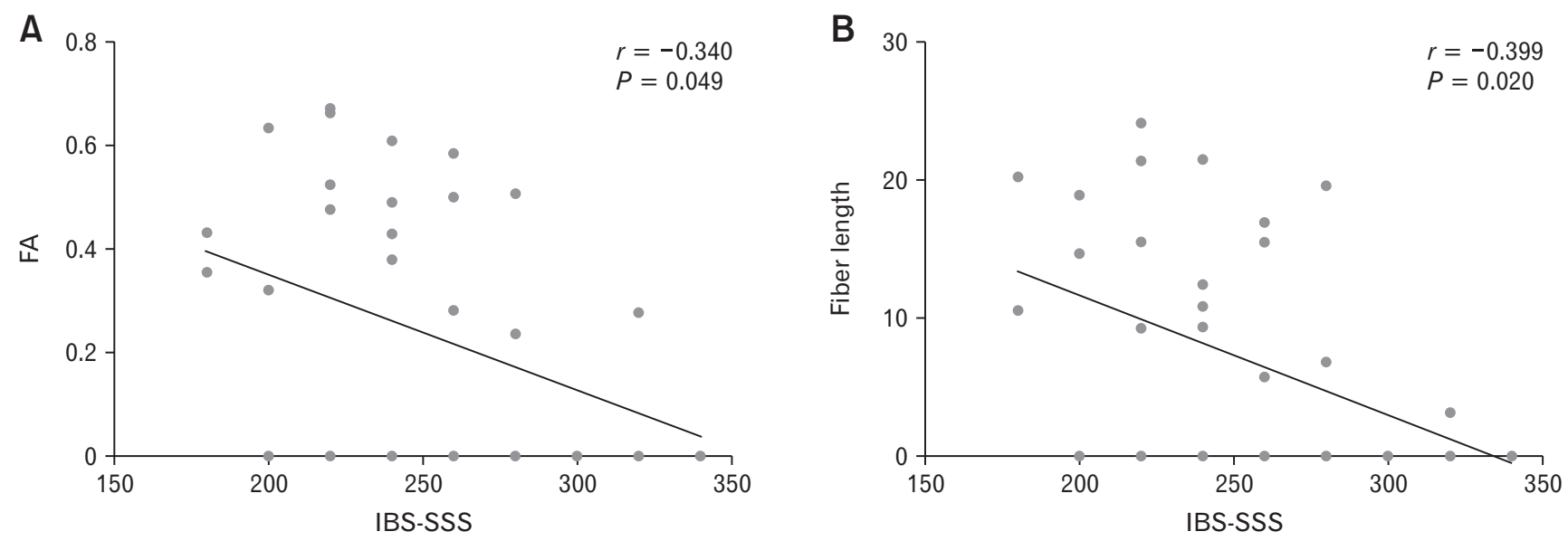

Figure 2. Correlation analyses between fractional anisotropy (FA)/fiber length and irritable bowel syndrome severity scoring system (IBS-SSS) score in posterior cingulate gyrus.

length, and fiber number. However, no significant differences were detected between IBS and HCs. As shown in Figure 2, we analyzed the correlations between FA/fiber length and IBS-SSS score using Pearson's correlation. The result showed that the FA $(r=-0.340, P<0.05)$ and fiber length $(r=-0.399, P<0.05)$ of the bilateral posterior cingulate gyrus showed significant negative correlation with the severity of IBS.

\section{Discussion}

In this study, we investigated the inter-hemispheric connection and structural asymmetry of IBS patients compared with HCs. Our results demonstrated that the $\mathrm{FC}$ of brain is the most sensitive to enteric disease, followed by anatomical connection, and the brain morphology of patients is the most stable. Compared with HCs, we found significant VMHC changes in the bilateral SOG, MOG, precuneus, PCG, SMA, and ANG. ROIs with abnormal changes may be related to the symptom manifestation of patients. Furthermore, we also found a negative correlation between interhemispheric FC and IBS-SSS in MOG. Although the differences of structural connectivity and asymmetry were not statistically significant, negative correlation was observed between anatomical connection and IBS-SSS. To our knowledge, this study is the first research aimed to evaluate inter-hemispheric connectivity and morphology of the brain in IBS patients.

\section{Inter-hemispheric Functional Connectivity}

VMHC describes the strength of FC between symmetric interhemispheric voxels. As shown in Figure 2 and Table 2, compared with $\mathrm{HCs}$, inter-hemispheric FC significantly increased in precuneus, PCG, and ANG in IBS patients. Moreover, these areas are all important components of the DMN. An increase in VMHC means a significant increase in inter-hemispheric connectivity of IBS patients. Some previous studies interpreted higher VMHC as more inter-hemispheric coordinated processing among the corresponding neuronal communities. ${ }^{58}$ The increase of VMHC in IBS patients may be related to the strengthened neural activity in ROIs associated with pain modulation and emotional processing. It has been shown that increased connectivity can be regarded as a sign of functional reorganization in early subtle brain damage. ${ }^{59} \mathrm{FC}$ has also been proposed as an objective marker of the impact of a brain injury. ${ }^{60}$ Thus, significantly increased VMHC may reflect an early damage and reorganization of DMN in IBS patients. Topological reorganization of DMN have been reported in some previous studies of IBS. ${ }^{61,62}$ The DMN is known to be altered in pain diseases and to participate in cognitive and memory-related aspects of pain modulation, ${ }^{63}$ and is thought to be preferentially effected by the presence of chronic pain. ${ }^{64}$ The significant increase of PCS and SF-MPQ scores also confirms this. Meanwhile, cognitive function in adult patients with IBS was found to be changed compared to $\mathrm{HCs}$, especially in visuospatial episodic memory functioning, which is perhaps related to the cortisol levels. ${ }^{65}$ Beyond this, PCG also plays a role in processing self-relevant emotional information. One study reported that there is increased processing of sad memories in depression. ${ }^{66}$ Abdominal symptoms can affect the patients' mood disorder such as depression and anxiety through a bottom-up model with the GBA, but also psychological factors will influence motor functions, sensory threshold, and stress reactivity of the gut 
via vagal and sympathetic afferents (top-down model). ${ }^{67}$ Other studies have found IBS patients display an increased recognition memory to words with negative emotional connotation or gastrointestinal symptoms, ${ }^{68}$ which may mean that selective attention to gastrointestinal symptoms play a role in decreased pain thresholds and consequently increased symptom severity.

The precuneus in humans is functionally connected with the motor, sensorimotor, and visual cortices. ${ }^{69}$ Our findings indicated that IBS patients showed increased inter-hemispheric FC in SOG and MOG. Meanwhile, as the severity increases, the VMHC of MOG is lower (Table 3). The SOF and MOF are near BA18 and BA19 that participate in various sensory inputs to the brain, such as somatosensory, visual, and auditory. ${ }^{70}$ In addition to this, VMHC was significantly decreased in the SMA, which has been reported to be activated by pain and play an important role in functional processing of painful stimuli. A prior functional study focusing on intrinsic whole brain FC pattern reported higher long-range FC density in the occipital lobe and right SMA in patients with IBS. ${ }^{71}$ Therefore, chronic abdominal pain may potentially affect the pain processing of SMA, which cause the abnormal FCs in these areas.

\section{Inter-hemispheric Anatomical Connectivity}

Although no group differences were found in the structural asymmetry and inter-hemispheric anatomical connections, FA and fiber length were negatively related with the severity in PCG (Fig. 1). We speculate that the anatomical connection of IBS patients would be damaged, but the differences did not reach statistical significance. Several studies have reported microstructural white matter abnormalities in IBS patients, ${ }^{33,72}$ but the region with significant differences were not consistent, including insula, thalamus, basal ganglia, and sensory/motor association/integration regions. ${ }^{33}$ Our experimental results showed that the changes in brain structure have not occurred, which would also suggest the structure of the brain is not easily attacked by functional gastrointestinal disorders.

Numerous factors can clearly affect the GBA in each process, and bi-directional communication can together add significant complexity to the system. Bilateral brain abnormalities in IBS patients were analyzed through inter-hemispheric connectivity and structural asymmetry. Our results reflected that the FC is often more vulnerable to IBS than structural connection, and the morphology of brain is the most insensitive. Similar results regarding inter-hemispheric connectivity was reported in previous studies, ${ }^{73,74}$ namely that the structural connectivity is relative stable.

In addition to these, we analyzed several common clinical scoring scales. As shown in Table 1, patients with IBS have significantly higher HAMD and HAMA scores compared with healthy adults, and the scores reached to mild depression $(8<$ HAMD $<13)$ and anxiety criteria (HAMA < 17), The results showed that patients with IBS are particularly susceptible to depressed or anxiety moods, which is consistent with the result of previous studies. ${ }^{7,75,76}$ In this regard, stress-related emotion can exacerbate gastrointestinal symptoms via the GBA, as well as affecting the QOL. ${ }^{77,78}$ Consistent with our speculation, statistical analysis results of IBS-QOL scores indicated the life quality declining of IBS patients. Gastrointestinal and psychological changes can interact and affect one another through the feedback mechanisms. Concomitantly, some clinical studies revealed that antidepressants have a continuous outcome for improvement of abdominal pain. ${ }^{79,80}$ Although it is unrealistic to treat IBS with psychotropic drugs, the abdominal pain of patients can be partially relieved after psychotherapeutic interventions. By reducing signaling and enhancing downregulation at the level of the dorsal horn, noxious painful experiences can be reduced. ${ }^{81}$

\section{Limitations}

Limitations of the current study must be recognized. First, this study only considered a Chinese population, and the sample size was relatively small. It is well known that some variables may have no statistically significant differences between the 2 groups because of the small sample size, and the performance of correlation analysis would be affected. ${ }^{82}$ Second, the use of cross-sectional data limits the interpretation, thus we cannot explore the causal relationship between brain alterations and intestinal symptoms. Third, the medication status of patients with IBS were not considered here, because different drugs or treatment modalities may affect the bidirectional brain-gut interactions. Consequently, future research may consider utilizing longitudinal imaging and enlarge the sample size to follow changes with symptom severity. Besides, inclusion of gut microbiota, serum, or stool metabolites in the analysis may further help us understand the pathological mechanisms of IBS.

\section{Conclusion}

In this study, through taking comprehensive analysis of the bilateral brain in patients with IBS, we speculate that inter-hemispheric FC is more vulnerable to IBS than anatomical connectivity, while the structural morphology of brain is the most stable. Meanwhile, the affected areas were concentrated in DMN and sensorimotor network. The results of our study are only preliminary, but it may provide theoretical basis for future research on the regulation of GBA and pathophysiology in functional intestinal diseases. 
Financial support: This work was supported in part by the National Key Research and Development Program of China (Grant No. 2019YFA0706200), in part by the National Natural Science Foundation of China (Grant No. 61632014, No. 61627808, No. 61210010), in part by the National Basic Research Program of China (973 Program, Grant No. 2014CB744600), in part by the Program of Beijing Municipal Science and Technology Commission (Grant No. Z171100000117005), in part by the Fundamental Research Funds for the Central Universities (Grant No. lzuxxxy2019-tm09), and in part by the Cuiying Scientific and Technological Innovation Program of Lanzhou University Second Hospital (Grant No. CY2018-QN03).

\section{Conflicts of interest: None.}

Author contributions: Guangyao Liu, Shan Li, Dekui Zhang, Zhijun Yao, and Bin Hu were involved in study conception and design; Hong Liu and Jie Feng recruited patients and collected the data; Nan Chen, Ziyang Zhao, and Man Guo analyzed the data; Guangyao Liu and Shan Li wrote the manuscript; all authors were involved in the discussion of study result and critical revision of the manuscript, and approved the final draft of this paper, including the authorship list.

\section{References}

1. Rodiño-Janeiro BK, Vicario M, Alonso-Cotoner C, Pascua-García R, Santos J. A review of microbiota and irritable bowel syndrome: future in therapies. Adv Ther 2018;35:289-310.

2. Lovell RM, Ford AC. Global prevalence of and risk factors for irritable bowel syndrome: a meta-analysis. Clin Gastroenterol Hepatol 2012;10:712-721, e4.

3. Sperber AD, Dumitrascu D, Fukudo S, et al. The global prevalence of IBS in adults remains elusive due to the heterogeneity of studies: a rome foundation working team literature review. Gut 2017;66:1075-1082.

4. Liu J, Hou X. A review of the irritable bowel syndrome investigation on epidemiology, pathogenesis and pathophysiology in China. J Gastroenterol Hepatol 2011;26(suppl 3):88-93.

5. Heitkemper M, Jarrett M, Bond EF, Chang L. Impact of sex and gender on irritable bowel syndrome. Biol Res Nurs 2003;5:56-65.

6. El-Salhy M. Irritable bowel syndrome: diagnosis and pathogenesis. World J Ggastroenterol 2012;18:5151-5163.

7. Fond G, Loundou A, Hamdani N, et al. Anxiety and depression comorbidities in irritable bowel syndrome (IBS): a systematic review and metaanalysis. Eur Arch Psychiatry Clin Neurosci 2014;264:651-660.

8. Spiller R, Humes D, Campbell E, et al. The patient health questionnaire 12 somatic symptom scale as a predictor of symptom severity and consulting behaviour in patients with irritable bowel syndrome and symptomatic diverticular disease. Aliment Pharmacol Ther 2010;32:811-820.

9. Gracie DJ, Hamlin JP, Ford AC. Longitudinal impact of IBS-type symptoms on disease activity, healthcare utilization, psychological health, and quality of life in inflammatory bowel disease. Am J Gastroenterol 2018;113:702-712.

10. Lee C, Doo E, Choi JM, et al. The increased level of depression and anxiety in irritable bowel syndrome patients compared with healthy controls: systematic review and meta-analysis. J Neurogastroenterol Motil 2017;23:349-362.

11. Carabotti M, Scirocco A, Maselli MA, Severi C. The gut-brain axis: interactions between enteric microbiota, central and enteric nervous systems. Ann Gastroenterol 2015;28:203-209.

12. Collins SM, Surette M, Bercik P. The interplay between the intestinal microbiota and the brain. Nat Rev Microbiol 2012;10:735-742.

13. Cryan JF, Dinan TG. Mind-altering microorganisms: the impact of the gut microbiota on brain and behaviour. Nat Rev Neurosci 2012;13:701712.

14. Lydiard RB, Pitrosky B, Hackett D, White C. Impact of gastrointestinal symptom severity on response to venlafaxine extended-release in patients with generalized anxiety disorder. J Clin Psychiatry 2004;65:838-844.

15. Lach G, Schellekens H, Dinan TG, Cryan JF. Anxiety, depression, and the microbiome: a role for gut peptides. Neurotherapeutics 2018;15:3659 .

16. Mayer EA, Padua D, Tillisch K. Altered brain-gut axis in autism: comorbidity or causative mechanisms? Bioessays 2014;36:933-939.

17. Zheng W, Woo C-W, Yao Z, et al. Pain-evoked reorganization in functional brain networks. Cereb Cortex 2020;30:2804-2822.

18. Schmaal L, Hibar DP, Sämann PG, et al. Cortical abnormalities in adults and adolescents with major depression based on brain scans from 20 cohorts worldwide in the ENIGMA major depressive disorder working group. Mol Psychiatry 2017;22:900-909.

19. Liu F, Guo W, Fouche JP, et al. Multivariate classification of social anxiety disorder using whole brain functional connectivity. Brain Struct Funct 2015;220:101-115.

20. Kim K, Shin JH, Myung W, et al. Deformities of the globus pallidus are associated with severity of suicidal ideation and impulsivity in patients with major depressive disorder. Sci Rep 2019;9:7462.

21. Davidson RJ, McEwen BS. Social influences on neuroplasticity: stress and interventions to promote well-being. Nat Neurosci 2012;15:689695.

22. Baliki MN, Schnitzer TJ, Bauer WR, Apkarian AV. Brain morphological signatures for chronic pain. PLoS One 2011;6:e26010.

23. Zhou D, Lebel C, Evans A, Beaulieu C. Cortical thickness asymmetry from childhood to older adulthood. Neuroimage 2013;83:66-74.

24. Rentería ME. Cerebral asymmetry: a quantitative, multifactorial, and plastic brain phenotype. Twin Res Hum Genet 2012;15:401-413.

25. Suárez R, Gobius I, Richards LJ. Evolution and development of interhemispheric connections in the vertebrate forebrain. Front Hum Neurosci 2014;8:497.

26. Jiang X, Shen Y, Yao J, et al. Connectome analysis of functional and structural hemispheric brain networks in major depressive disorder. Transl Psychiatry 2019;9:136. 
27. Jung YH, Shin JE, Lee YI, Jang JH, Jo HJ, Choi SH. Altered amygdala resting-state functional connectivity and hemispheric asymmetry in patients with social anxiety disorder. Front Psychiatry 2018;9:164.

28. Zuo Z, Ran S, Wang Y, et al. Asymmetry in cortical thickness and subcortical volume in treatment-naïve major depressive disorder. Neuroimage Clin 2019;21:101614.

29. Guleria A, Karyampudi A, Singh R, et al. Mapping of brain activations to rectal balloon distension stimuli in male patients with irritable bowel syndrome using functional magnetic resonance imaging. J Neurogastroenterol Motil 2017;23:415-427.

30. Nan J, Zhang L, Chen Q, et al. White matter microstructural similarity and diversity of functional constipation and constipation-predominant irritable bowel syndrome. J Neurogastroenterol Motil 2018;24:107-118.

31. Liu X, Silverman A, Kern M, et al. Excessive coupling of the salience network with intrinsic neurocognitive brain networks during rectal distension in adolescents with irritable bowel syndrome: a preliminary report. Neurogastroenterol Motil 2016;28:43-53.

32. Qi R, Liu C, Ke J, et al. Abnormal amygdala resting-state functional connectivity in irritable bowel syndrome. AJNR Am J Neuroradiol 2016;37:1139-1145.

33. Ellingson BM, Mayer E, Harris RJ, et al. Diffusion tensor imaging detects microstructural reorganization in the brain associated with chronic irritable bowel syndrome. Pain 2013;154:1528-1541.

34. Piché M, Chen JI, Roy M, Poitras P, Bouin M, Rainville P. Thicker posterior insula is associated with disease duration in women with irritable bowel syndrome (IBS) whereas thicker orbitofrontal cortex predicts reduced pain inhibition in both IBS patients and controls. J Pain 2013;14:1217-1226.

35. Hubbard CS, Becerra L, Heinz N, et al. Abdominal pain, the adolescent and altered brain structure and function. PLoS One 2016;11:e0156545.

36. Blankstein U, Chen J, Diamant NE, Davis KD. Altered brain structure in irritable bowel syndrome: potential contributions of pre-existing and disease-driven factors. Gastroenterology 2010;138:1783-1789.

37. Seminowicz DA, Labus JS, Bueller JA, et al. Regional gray matter density changes in brains of patients with irritable bowel syndrome. Gastroenterology 2010;139:48-57, e42.

38. Meleine M, Matricon J. Gender-related differences in irritable bowel syndrome: potential mechanisms of sex hormones. World J Gastroenterol 2014;20:6725-6743.

39. Drossman DA. Functional gastrointestinal disorders: history, pathophysiology, clinical features, and rome IV. Gastroenterology 2016;150:12621279, e2.

40. Clinical guidelines on the identification, evaluation, and treatment of overweight and obesity in adults--the evidence report. National Institutes of Health. Obes Res 1998;6(suppl 2):51S-209S.

41. Francis CY, Morris J, Whorwell PJ. The irritable bowel severity scoring system: a simple method of monitoring irritable bowel syndrome and its progress. Aliment Pharmacol Ther 1997;11:395-402.

42. Sullivan MJ, Bishop SR, Pivik J. The pain catastrophizing scale: development and validation. Psychol Assess 1995;7:524.

43. Kroenke K, Spitzer RL, Williams JB. The PHQ-15: validity of a new measure for evaluating the severity of somatic symptoms. Psychosom
Med 2002;64:258-266.

44. Melzack R. The short-form McGill pain questionnaire. Pain 1987;30:191-197.

45. Hamilton M. Hamilton anxiety scale. Group 1959;1:4. Available from URL: https://www.blix.uzh.ch/PDF/hama.pdf (accessed 24 Jun 2021).

46. Hamilton M. A rating scale for depression. J Neurol Neurosurg Psychiatry 1960;23:56-62.

47. Patrick DL, Drossman DA, Frederick IO, DiCesare J, Puder KL. Quality of life in persons with irritable bowel syndrome: development and validation of a new measure. Dig Dis Sci 1998;43:400-411.

48. Chao-Gan Y, Yu-Feng Z. DPARSF: a MATLAB toolbox for "pipeline" data analysis of resting-state fMRI. Front Syst Neurosci 2010;4:13.

49. Friston KJ, Holmes AP, Worsley KJ, Poline JP, Frith CD, Frackowiak RS. Statistical parametric maps in functional imaging: a general linear approach. Hum Brain Mapp 1994;2:189-210.

50. Dale AM, Fischl B, Sereno MI. Cortical surface-based analysis. I. Segmentation and surface reconstruction. Neuroimage 1999;9:179-194.

51. Fischl B, Dale AM. Measuring the thickness of the human cerebral cortex from magnetic resonance images. Proc Natl Acad Sci USA 2000;97:11050-11055.

52. Fischl B, Salat DH, Busa E, et al. Whole brain segmentation: automated labeling of neuroanatomical structures in the human brain. Neuron 2002;33:341-355.

53. Cui Z, Zhong S, Xu P, He Y, Gong G. PANDA: a pipeline toolbox for analyzing brain diffusion images. Front Hum Neurosci 2013;7:42.

54. Smith SM, Jenkinson M, Woolrich MW, et al. Advances in functional and structural MR image analysis and implementation as FSL. Neuroimage 2004;23(suppl 1):S208-S219.

55. Wang R, Benner T, Sorensen AG, Wedeen VJ. Diffusion toolkit: a software package for diffusion imaging data processing and tractography. In Proc Intl Soc Mag Reson Med, Berlin 2007.

56. Zuo XN, Kelly C, Di Martino A, et al. Growing together and growing apart: regional and sex differences in the lifespan developmental trajectories of functional homotopy. J Neurosci 2010;30:15034-15043.

57. Song XW, Dong ZY, Long XY, et al. REST: a toolkit for resting-state functional magnetic resonance imaging data processing. PLoS One 2011;6:e25031.

58. Fan H, Yang X, Zhang J, Chen Y, Li T, Ma X. Analysis of voxel-mirrored homotopic connectivity in medication-free, current major depressive disorder. J Affect Disord 2018;240:171-176.

59. van Duinkerken E, Schoonheim MM, Sanz-Arigita EJ, et al. Restingstate brain networks in type 1 diabetic patients with and without microangiopathy and their relation to cognitive functions and disease variables. Diabetes 2012;61:1814-1821.

60. Castellanos NP, Bajo R, Cuesta P, et al. Alteration and reorganization of functional networks: a new perspective in brain injury study. Front Hum Neurosci 2011;5:90.

61. Qi R, Ke J, Schoepf UJ, et al. Topological reorganization of the default mode network in irritable bowel syndrome. Mol Neurobiol 2016;53:6585-6593.

62. Letzen JE, Craggs JG, Perlstein WM, Price DD, Robinson ME. Functional connectivity of the default mode network and its association with 
pain networks in irritable bowel patients assessed via lidocaine treatment. J Pain 2013;14:1077-1087.

63. Baliki MN, Mansour AR, Baria AT, Apkarian AV. Functional reorganization of the default mode network across chronic pain conditions. PLoS One 2014;9:e106133.

64. Farmer MA, Baliki MN, Apkarian AV. A dynamic network perspective of chronic pain. Neurosci Lett 2012;520:197-203.

65. Kennedy PJ, Clarke G, O'Neill A, et al. Cognitive performance in irritable bowel syndrome: evidence of a stress-related impairment in visuospatial memory. Psychol Med 2014;44:1553-1566.

66. Cheng W, Rolls ET, Qiu J, et al. Increased functional connectivity of the posterior cingulate cortex with the lateral orbitofrontal cortex in depression. Transl Psychiatry 2018;8:90.

67. Stasi C, Rosselli M, Bellini M, Laffi G, Milani S. Altered neuro-endocrine-immune pathways in the irritable bowel syndrome: the top-down and the bottom-up model. J Gastroenterol 2012;47:1177-1185.

68. Gibbs-Gallagher N, Palsson OS, Levy RL, Meyer K, Drossman DA, Whitehead WE. Selective recall of gastrointestinal-sensation words: evidence for acognitive-behavioral contribution to irritable bowel syndrome. Am J Gastroenterol 2001;96:1133-1138.

69. Del Campo N, Payoux P, Djilali A, et al. Relationship of regional brain beta-amyloidto gait speed. Neurology 2016;86:36-43.

70. Chen L, Li C, Zhai J, et al. Altered resting-state signals in patients with acute stroke in or under the thalamus. Neurosci Bull 2016;32:585-590.

71. Weng Y, Qi R, Liu C, et al. Disrupted functional connectivity density in irritable bowel syndrome patients. Brain Imaging Behav 2017;11:18121822.

72. Chen JY, Blankstein U, Diamant NE, Davis KD. White matter abnormalities in irritable bowel syndrome and relation to individual factors. Brain Res 2011;1392:121-131.

73. Wu Y, Ji GJ, Li K, et al. Interhemispheric connectivity in drug-naive be- nign childhood epilepsy with centrotemporal spikes: combining function and diffusion MRI. Medicine 2015;94:e1550.

74. Qi R, Liu C, Weng Y, et al. Disturbed interhemispheric functional connectivity rather than structural connectivity in irritable bowel syndrome. Front Mol Neurosci 2016;9:141.

75. Hartono JL, Mahadeva S, Goh KL. Anxiety and depression in various functional gastrointestinal disorders: do differences exist? J Dig Dis 2012;13:252-257.

76. Lee SP, Sung IK, Kim JH, Lee SY, Park HS, Shim CS. The effect of emotional stress and depression on the prevalence of digestive diseases. J Neurogastroenterol Motil 2015;21:273283.

77. Cho SH, Park JM, Lim CH, et al. Anxiety, depression and quality of life in patients with irritable bowel syndrome. Gut Liver 2011;5:29-36.

78. Karakoyun-Celik O, Gorken I, Sahin S, Orcin E, Alanyali H, Kinay M. Depression and anxiety levels in woman under follow-up for breast cancer: relationship to coping with cancer and quality of life. Med Oncol 2010;27:108-113.

79. Ruepert L, Quartero AO, de Wit NJ, van der Heijden GJ, Rubin G, Muris JW. Bulking agents, antispasmodics and antidepressants for the treatment of irritable bowel syndrome. Cochrane Database Syst Rev 2011:CD003460.

80. Ford AC, Lacy BE, Harris LA, Quigley EMM, Moayyedi P. Effect of antidepressants and psychological therapies in irritable bowel syndrome: an updated systematic review and meta-analysis. Am J Gastroenterol 2019;114:21-39.

81. Dekel R, Drossman DA, Sperber AD. The use of psychotropic drugs in irritable bowel syndrome. Expert Opin Investig Drugs 2013;22:329339.

82. Ioannidis JP. Why most published research findings are false. PLoS Med 2005;2:e124. 